\title{
Is a matched sibling the ideal donor for hematopoietic cell transplant?
}

\author{
Mary Eapen
}

Department of Medicine, Medical College of Wisconsin, Milwaukee, WI, USA

E-mail:meapen@mcw.edu

doi:10.3324/haematol.2018.196980

I $\mathrm{n}$ this issue of Haematologica, Salvatore and colleagues compare outcomes after transplantation of grafts from a haploidentical relative or a HLA-matched sibling for adults with acute myeloid leukemia in first complete remission. ${ }^{1}$ They conclude survival was inferior after transplantation of peripheral blood or bone marrow from a haploidentical relative compared to an HLA-matched sibling for patients with intermediate-risk cytogenetics. On the other hand, for patients with high-risk cytogenetics, survival did not differ by donor type despite a non-significant reduction in relapse risk after haploidentical transplant. Non-relapse mortality risks were higher after haploidentical transplantation negated any survival advantage to be expected with the modest reduction in relapse after haploidentical transplantation for AML with high-risk cytogenetics. The observation of a reduction in relapse risk, albeit non-significant, after haploidentical transplantation is intriguing. While it is tempting to attribute this to an enhanced graft-versus-host leukemia effect in the setting of an HLA-mismatched transplant, the reduction in relapse risk was only seen for patients with high-risk cytogenetics. Can this be explained by differences in transplant conditioning regimen intensity? The study population received both myeloablative and reduced intensity transplant conditioning regimens. Among patients with intermediate risk cytogenetics, reduced intensity conditioning was associated with higher relapse. Yet, in the group of patients with high-risk cytogenetics, relapse risks did not differ by transplant conditioning regimen intensity leading us to conclude this merits further investigation.

These data raise a fundamental question: when should we select an HLA-mismatched relative instead of an HLAmatched sibling? If an HLA-matched sibling is medically unfit or unwilling to donate, an HLA-mismatched relative could be the obvious choice for a number of reasons including, but not limited to, the ease of availability of the donor and timing of transplantation. Yet, when an HLA-matched sibling is medically fit and willing to donate are there circumstances that warrant selection of a haploidentical relative? A recent joint report from the European Society for Blood and Marrow Transplant and the Center for International Blood and Marrow Transplant explored whether post-transplant cyclophosphamide can nullify the detrimental effect of HLA mismatch for acute myeloid and lymphoblastic leukemia. ${ }^{2}$ The report showed haploidentical siblings donated to adult patients younger than 55 years and offspring donated to those 55 years and older. After adjusting for risk factors associated with survival the study concluded an HLA-matched sibling was a better choice than an offspring in patients 55 years and older. In the group with patients aged 18-54 years, a comparison of haploidentical to HLA-matched sibling transplant did not reveal differences in survival. The characteristics of the patients studied in two reports and their numbers differ ${ }^{1,2}$ and this is the most likely explanation for the differences between the two reports. As the report by Salvatore and colleagues did not consider donor-recipient relationship, we do not know whether the effect of cytogenetic risk on survival may be explained by donor-recipient relationship and patient age on survival. However, both these reports present more questions in regards to donor selection.

Donor age is associated with survival after unrelated donor transplantation. ${ }^{3}$ Survival is better after transplantation of grafts from younger donors after adjustment for donor-recipient HLA-match. Donor age is challenging to study in the setting of HLA-matched sibling transplants as generally the age of siblings falls within the same decade. Others have compared transplantation of grafts from a young unrelated donor and an HLA-matched sibling in older adults with hematologic malignancy and confirm there is no survival advantage when a young unrelated donor is chosen in favor of an older HLA-matched sibling. ${ }^{4}$ So, is there a potential advantage to selecting an offspring who is likely to be about 2-3 decades younger than the parent? The effects of donor age on adults with hematologic malignancy undergoing haploidentical transplantation has been studied by others. ${ }^{5}$ In that report, the age of the patient ( $\geq 55$ years) rather than the age of the donor was associated with higher mortality. ${ }^{5}$ The study did not identify any donor factors that were associated with mortality. ${ }^{5}$ It is worth noting that the numbers of haploidentical transplants available for study are modest when compared to the numbers of HLA-matched sibling and unrelated donor transplants. Therefore, with the increasing numbers of haploidentical transplants performed, it is incumbent upon the community of transplant physicians to carefully evaluate the effects of characteristics of haploidentical donors on transplant outcomes.

Lastly, how can we best study donor selection for hematopoietic cell transplant? There is general agreement that when treatment options are being studied, a randomized trial is the gold standard. Planning and executing randomized trials is more easily said than done. In the context of related donor transplantation, subjects must have an HLAmatched sibling and a haploidentical relative for randomization. This in itself is limiting, as several more subjects will have a suitable haploidentical relative rather than an HLAmatched sibling. Secondly, we do not know whether there are differences amongst the haploidentical relatives and should randomization consider donor-recipient relationship. Thirdly, are physicians willing to randomize patients with an HLA-matched sibling to receive a haploidentical relative? While some may not, others may find this unacceptable. Regardless of the complexities of conducting randomized trials there is no denial in the lengthy duration of these trials and the expense incurred. Hence, there is reliance on data collected by large transplant registries to better understand the effect of donor types on transplant outcomes. In the meantime, the report by Salvatore and colleagues compels us to select an HLA-matched sibling when such a donor is 
available. A haploidentical relative is a suitable alternative when an HLA-matched sibling is not available.

\section{References}

1. Salvatore D, Labopin M, Ruggeri A, et al. Outcomes of hematopoietic stem cell transplantation from unmanipulated haploidentical versus matched sibling donor in patients with acute myeloid leukemia in first complete remission with intermediate or high-risk cytogenetics: a study from the Acute Leukemia Working Party of the European Society for Blood and Marrow Transplantation. Haematologica. 2018;103(8): 1317-1328.
2. Robinson TM, Fuchs EJ, Zhang MJ, et al. Related donor transplants: has posttransplant cyclophosphamide nullified the detrimental effect of HLA mismatch? Blood Adv. 2018;2(11):1180-1186.

3. Kollman C, Spellman SR, Zhang MJ, et al. The effect of donor characteristics on survival after unrelated donor transplantation for hematologic malignancy. Blood. 2016;127(2):260-267.

4. Alousi AM, Le-Rademacher J, Saliba RM, et al. Who is the better donor older hematopoietic transplant recipients: an older-aged sibling or a young, matched unrelated volunteer? Blood. 2013;121(13): 2567-2573.

5. McCurdy SR, Zhang MJ, St. Martin A, et al. Effect of donor characteristics on haploidentical transplantation with posttransplant cyclophosphamide. Blood Adv. 2018;2(3):299-307.

\title{
G-protein coupled receptor (GPCR) mutations in lymphoid malignancies: linking immune signaling activation and genetic abnormalities
}

\author{
Jose Angel Martinez-Climent \\ Division of Hematological Oncology, Center for Applied Medical Research, University of Navarra, IDISNA, CIBERONC, \\ Pamplona, Spain
}

E-mail: jamcliment@unav.es

doi:10.3324/haematol.2018.196998

M arginal-zone B-cell lymphomas of mucosa-associated lymphoid tissue (MALT) arise from a background of chronic microbial infections or autoimmune disorders at diverse extranodal sites. ${ }^{1,2}$ The best characterized examples are gastric MALT lymphoma following Helicobacter pylori infection, and salivary gland or thyroid MALT lymphomas developing in patients with Sjögren syndrome or Hashimoto thyroiditis, respectively., It is now accepted that such chronic microenvironmental inflammation stimulates surface BCR, TLR and CD40 receptors in B lymphocytes that converge to activate downstream NF- $\mathrm{KB}$ signaling, which leads to the local expansion of autoreactive $B$ cells eventually suffering malignant transformation through the acquisition of genetic changes. ${ }^{5}$ Among them, three hallmark chromosomal translocations, $\mathrm{t}(11 ; 18)(\mathrm{q} 21 ; \mathrm{q} 21), \mathrm{t}(14 ; 18)(\mathrm{q} 32 ; \mathrm{q} 21)$ and $\mathrm{t}(1 ; 14)(\mathrm{p} 22 ; \mathrm{q} 32)$, play a major part in MALT lymphoma origination through dysregulating MALT1 enzymatic activity that constitutively triggers the NF- $\mathrm{\kappa B}$ pathway independently of antigenic stimuli.-9 Other recurrent mutations in the MYD88, TBL1XR1, KLF2 and TNFAIP3 genes are similarly a consequence of chronic receptor stimulation and further promote NF- $\mathrm{kB}$ signaling, contributing to lymphoma transformation..$^{10} \mathrm{~A}$ second signaling pathway recurrently found to be involved in marginalzone lymphoma (MZL) pathogenesis is $\mathrm{NOTCH}$, primarily including mutations in the C-terminal PEST domain of NOTCH2 and NOTCH1 genes that enhance the stability of intracellular protein domains after being triggered by microenvironmental interactions. ${ }^{5}$ Thus, both the active chronic immunological stimuli and the acquired genetic abnormalities have critical roles during the development of MALT lymphoma through dysregulating similar molecular mechanisms.

In this issue of the Journal, Moody et al. expand this intriguing oncogenic co-operation between immune receptor signaling and genetic abnormalities in MALT lymphoma. They report the discovery of somatic mutations in the G-protein coupled receptors (GPCRs) GPR34 and CCR6 not previously reported in human malignancies. ${ }^{11}$ The Authors performed whole exome sequencing of 21 salivary gland and thyroid tumors, and also carried out sequencing analysis of 249 MALT lymphomas, to define distinct mutation profiles in tumors of various sites. Those of the salivary gland were characterized by frequent TBL1XR1 and GPR34 mutations, whereas CCR6 changes were found in MALT lymphomas at different locations. The majority of GPR34 and CCR6 mutations clustered in the cytoplasmic tail, potentially leading to truncated gain-of-function proteins enabling constitutive ligand-dependent receptor activation. ${ }^{12}$ Thus, a novel synergistic mechanism between constitutively active NF- $\mathrm{KB}$ and GPCR signaling pathways is proposed to participate in the development of MALT lymphoma (Figure 1A).

G-protein coupled receptors are made up of a large superfamily of cell surface ligands that regulate and transmit extracellular signals across the plasma membrane to induce a range of cellular and physiological responses. Despite this diversity, however, their structure, activation, signaling and regulatory mechanisms are remarkably conserved. GPCRs contain seven transmembrane spanning $\alpha$ helices linked by three intracellular and three extracellular loop regions, an extracellular amino-terminal domain, and an intracellular carboxyl tail. In response to ligand binding, the receptor undergoes conformational changes to couple and activate heterotrimeric $G$ proteins $(G \alpha, G \beta$ and $G \gamma)$ at the plasma membrane that regulate downstream signaling effectors. To turn off the response, GPCR kinases are recruited to phosphorylate the receptor and prepare them for $\beta$-arrestin binding, which compete with $G$ protein coupling and desensitize the G-protein-mediated signaling response. ${ }^{13}$ Aberrant receptor activity has been shown in 